DOI: https://doi.org/10.24297/jssr.v15i.8670

\title{
Methods and Rule-Of-Thumbs in The Determination of Minimum Sample Size When Appling Structural Equation Modelling: A Review
}

\author{
Ranatunga RVSPK ${ }^{1}$, Priyanath $\mathrm{HMS}^{2}$, Megama RGN ${ }^{3}$ \\ ${ }^{1}$ Centre for Computer Studies, Sabaragamuwa University of Sri Lanka, Belihuloya. Sri Lanka. \\ ${ }^{2}$ Department of Economics and Statistics, Sabaragamuwa University of Sri Lanka, Belihuloya. Sri Lanka. \\ ${ }^{3}$ Faculty of Applied Sciences, University of Sri Jayewardenepura. Nugegoda. Sri Lanka. \\ priya@ssl.sab.ac.lk
}

\begin{abstract}
Basic methods and techniques involved in the determination of minimum sample size at the use of Structural Equation Modeling (SEM) in a research project, is one of the crucial problems faced by researchers since there were some controversy among scholars regarding methods and rule-of-thumbs involved in the determination of minimum sample size when applying Structural Equation Modeling (SEM). Therefore, this paper attempts to make a review of the methods and rule-of-thumbs involved in the determination of sample size at the use of SEM in order to identify more suitable methods. The paper collected research articles related to the sample size determination for SEM and review the methods and rules-of-thumb employed by different scholars. The study found that a large number of methods and rule-of-thumbs have been employed by different scholars. The paper evaluated the surface mechanism and rules-of-thumb of more than twelve previous methods that contained their own advantages and limitations. Finally, the study identified two methods that are more suitable in methodologically and technically which have identified by non-robust scholars who deeply addressed all the aspects of the techniques in the determination of minimum sample size for SEM analysis and thus, the prepare recommends these two methods to rectify the issue of the determination of minimum sample size when using SEM in a research project.
\end{abstract}

Keywords: Minimum Sample Size Determination, Structural Equation Modelling.

\section{Introduction}

The Structural Equation Model (SEM) is one of the most extensively used quantitative multivariate data analysis technique which is currently employing to examine the relationship between observed and latent variables of the exploratory, and confirmatory hypothesis testing approaches as well as various types of predictive analysis models. This modeling technique is particularly suitable in the social sciences where mostly the key concepts are not openly observable and are inherently latent generally defined as latent variables (Kline, 1998; Kock \& Lynn, 2012). The SEM is the prominent approach to analysing the path models with such latent variables to produce the final conclusions about the nature of combining the theories. SEM has its roots in path analysis, which was invented by the geneticist Sewall Wright in 1921 (as cited by Hox \& Bechger, 1999). As mentioned by Westland (2010), this modeling technique has been developed in three different streams, such as equation regression methods, iterative maximum likelihood algorithms for path analysis, and iterative least-squares fit algorithms for path analysis.

SEM has greater flexibility on its nature because it can be used to examine very complex relationships among a variety of data types such as dimensional, categorical censored or count as well can be used to compare among the alternative models (Schreiber, Nora, Stage, Barlow, \& King, 2006; Wolf, Harrington, Clark, \& Miller, 2013). Conversely, inside of this flexibility of the SEM, it has been identified an anomaly, which is the unavailability of comprehensive guidelines regarding the sample size requirements by the past researchers. However, different 
researchers suggested and implemented different procedures as a rule-of-thumb for deciding sample size for their SEM-based researches (MacCallum \& Austin, 2000; Westland, 2010). Therefore, this paper aims to make a review of the basic concepts involved in the determination of the minimum sample size for SEM and identifies more suitable methods for the determination of the minimum sample size for SEM.

The paper organized as follows; first introduction with research problem and objectives, second briefly explained the theoretical background of the study. Used methodology has been mentioned thirdly, and results and discussion represent forth based on the review of the past literature. Finally, a summary and the conclusions are been included.

\section{Theoretical Background}

The researcher uses sampling because of the inability to study the population as required. Inadequate, or unnecessary sample sizes impact the quality and accuracy of research. Hence, it is very important to represent the characteristics of the population within the studied sample. One of the most important factors is the selection of the number of different cases from the population, which will represent all the population characteristics. Three criteria need to be specified to determine the appropriate sample size, such as the level of precision, the level of confidence, and the degree of variability in the attributes being measured (Miaoulis \& Michener, 1976). There are three bases to choose a sample size, such as cost base, variance base, and statistical power base (Singh \& Masuku, 2014). The statistical power base sample size determination is using a target for the power of a statistical test to be applied once the sample is collected where the quality of the resulting estimates and assessed based on the power of a hypothesis test are been used to judge the sample size (Singh \& Masuku, 2014).

The difference between the calculated sample parameters and the actual population parameters denoted by the error. According to Muthén \& Muthén, (2002), the precision depicts this nature, and the sample parameters should close to the population parameters with the narrow margin of errors, which means high precision. The power of statistics refers to the type II error or $1-\beta$, which means the probability of rejecting the false null hypothesis (Cohen, 1988). Mostly, the power measured as 0.8 or $80 \%$ of the probability of rejecting the null hypothesis is used by social science researches (Cohen, 1988; Goodhue, Lewis, \& Thompson, 2012). In the context of SEM, the power has been used to test the model mostly, the powerbase sample size determination is been utilized ((Goodhue et al., 2012; Kock \& Hadaya, 2018; Westland, 2010).

The decision taken by the researcher directly affects the validation of the study, suitability of parametric or nonparametric methods to use, as well as the precision and power of the model's parameter estimates. The American Psychological Association, (2009) mentioned that "how this intended sample size was determined (e.g., analysis of power or precision). If interim analysis and stopping rules were used to modify the desired sample size, describe the methodology and results". Wilkinson (1999) mentioned that the researchers should provide the process of sampling and the size as well as should document the effect size and the analytic procedure of the power calculation.

\section{Methodology}

The minimum sample size determination problem is not clearly defined, and still, it is being defined by the researchers. Thus, this study uses the exploratory study based on secondary information, where this method can be used to understand the existing problem more preciously. The study started with the general idea and employed it as a medium to identify issues that can be the focus of future researches. The research fundamentally used the grounded theory approach, and it based on more than fifty articles published in journals relevant to the determination of minimum sample size for SEM analysis. 


\section{Review Minimum Sample Size Determination}

Although the minimum sample size determination of SEM is more problematic various rules-of-thumb have been suggested in the SEM literature. Nunnally, (1967) mentioned that the sampling error in a $\beta$ weight is a function of all the variables used in the regression analysis. And he mentioned two facts. The former was a sampling error that is a function of sample size and their intercorrelation, as well as the latter, was systematic differences between the characteristics of the two samples. According to his idea even though the Regression weights may be robust across samples that have quite different means and variances, but this should not be taken for granted. Further, he noted that "as a rule of thumb, but not a magical number, you should have 10 subjects per predictor in order to even hope for a stable prediction equation" (Nunnally, 1967); Wolf et al., 2013). With this proposal, the debate of minimum sample size determination in the SEM has significantly evolved.

The method of minimum sample size 100 or 400 has been suggested by Boomsma (1982) and (1985). They further study suggested a ratio of indicators to latent variables as $r=p / k$. According to this rule, $r=4$ requires sample size at least 100 and $r=2$ requires 400 sample size. Further enhancement with 35000 Monte Carlo simulations has done by Marsh, Balla, \& McDonald (1988) of this rule and suggested if $r=3$ requires at least 200 sample size and $r=2$ needs at least 400 sample size as well as if it is $r=12$ then the sample size is small and at least 50 . According to the findings of these two studies, researchers attempted to use fewer indicators per latent variables for controlling the cost. Kline, (2005) mentioned this rule as $N \geq 100$ rule. A similar explanation has been presented by Ding, Velicer, \& Harlow (1995) concerned that most of the studies which evaluated the sample size on different fit indices of SEM found that most of the fit indices were biased under the small sample size. Anderson \& Gerbing, (1984) also mentioned a similar result and said it is more serious on the small sample size combined with other factors such as low loading size and a large ratio of indicators to factor. Similar studies such as Bearden, Sharma \& Teel (1982); Bentler, (1990); Bentler \& Bonett, (1980); Curran, West \& Finch (1996); Marsh, Balla \& McDonald (1988); and Mulaik, James, Alstine, Bennett, Lind, \& Stilwell (1989) implies the same results with different fit indices. Finally, Ding et al., (1995) recommended 100 to 150 minimum sample size for conducting the SEM according to these evaluations.

The number of free parameters in the model also considered determining the sample size (Raykov, 2006). According to this rule, the minimum sample size should be ten times the number of free parameters of the model. If the model has 20 free parameters then the number of observations should be 200. Bentler, (1990) suggested that a $5: 1$ ratio of a sample size to the number of free parameters. Further, Velicer \& Fava, (1998) reviewed the recommendations of past literature in the minimum sample determination and concluded minimum sample size is not a function of indicators. According to them the goodness of fit and obtain the proper solution achieved by two things such as a greater number of indicators per latent variable and higher factor lodgings in the given sample size. Consequently, MacCallum et al., (1999) argued and demonstrated that model characteristics such as the level of commonality across the variables, sample size, and degree of factor determinacy may influence to the parameter estimates and model fit statistics and hence, it makes some doubts on the above sample size rules-of-thumb to particular SEM analysis.

If the model is complex the PLS-SEM works efficiently in a smaller sample size (Fornell \& Bookstein, 1982). Goodhue, Lewis, \& Thompson $(2006,2007)$ tried to examine the rule of ten subjects by using the Monte Carlo simulation and made the comparison with sample sizes 40, 90, 150, and 200 under the effect size such as 'large', 'medium', 'small' and 'no effect'. According to their conclusions they mentioned "for simple SEM models with normally distributed data and relatively reliable measures, none of the techniques have adequate power to detect small or medium effects at small sample size" (Goodhue et al., 2006). Tanaka, (1987) suggested the sample size of the SEM model should depend on the number of estimated parameters rather than the tal number of indicators. However, Westland, (2010) claimed that since the present SEM models are typically estimated in their entirety, and number of unique entries in the covariance matrix is $(p(p+1)) / 2$ when $p$ is the number of indicators and it should be accepted as sample size is proportional to $(p(p+1)) / 2$ rather than $p$. Further, he mentioned this minimum sample size determination problem is more complex than the above and it has been shown by the Monte Carlo simulation studies done in the 1980s and 1990s. 
According to Ringle, Sarstedt, \& Straub (2012), it should be approximately eight constructs for a smaller sample. However, identification of the required minimum sample size for the PLS-SEM analysis is one of the fundamental and important issues which should be deeply considered by the researchers (Goodhue et al., 2012; Kock \& Hadaya, 2018). Hair, Ringle, \& Sarstedt (2011) introduced the "10-times rule" and it is the method that extensively used in the past literature (Kock \& Hadaya, 2018). This rule assumed that the sample size should be greater than 10 times the maximum number of inner or outer model links pointing at any latent variable in the entire model and consequently, this is the very simple method to estimate the sample size compared to the other methods. Thus, this is more popular among researchers. (Hair et al., 2011; Kock \& Hadaya, 2018; Westland, 2010). However, according to Goodhue et al., (2012) and Kock \& Hadaya, (2018) "10-times rule" method for estimation of minimum sample size lead to inaccurate estimates most of the time.

Monte Carlo simulation also has been employed as a method of determining the minimum sample size of the PLS-SME (Kock, 2016; Paxton et al., 2001; Robert \& Casella, 1999; Wolf et al., 2013). Knowledge of the sampling distribution is the key to the evaluation of the behaviour of a statistic and thus the Monte Carlo simulations provide facilities to the researchers for evaluating the fixed sampling performance of estimators by creating controlled conditions from which sampling distributions of parameter estimates are produced. Therefore, the researcher can artificially create and use the sampling distribution to decide the properties of the statis tics as well as its efficiency (Paxton et al., 2001). According to Kock \& Hadaya, (2018)"minimum sample size estimation in PLS-SEM requires the researcher to set a number of sample size points (e.g., 15, 20, 30 and 40), generate a number of samples (e.g., 1000) for each sample size point, calculate the percentages of samples in which significant effects (e.g., for which $\mathrm{P}<.05$ ) were found for each sample size point (the power associated with each sample size), and estimate via interpolation the minimum sample size at which power reaches the desired threshold (i.e., .8)" and they further mentioned that though the Monte Carlo simulation method is a prominent method for determining the minimum sample size it is a difficult way and for which both technical and methodological expertise with good computer programming skills is required as well as it is time-consuming (Kock \& Hadaya, 2018). Wolf et al., (2013) also concluded that "the final lesson learned is that determining sample size requirements for SEM necessitates careful, deliberate evaluation of the specific model at hand".

Hair et al., (2014) have discussed another alternative method instead of " 10 times rule" for minimum sample size estimation and Kock \& Hadaya, (2018) referred it as the "minimum R-squared method" since it uses minimum R2 in the model for estimating the minimum sample size. This method particularly has been built on Cohen, (1988) power table for least squares regression and three elements require for determining the sample size. The first element of the minimum R-squared method is the maximum number of arrows pointing at a latent variable in a model, used significance level is the second and third is the minimum $\mathrm{R}^{2}$ in the model. Table 01 illustrates the reduced version of the table presented by Hair et al., (2014) and it depends on the significance level of 0.05 , which is the most commonly used significance level and assumes that the power is set at 0.8 . This method appears to be an improvement over the 10-times rule method, as it takes as an input at least one additional element beyond the network of links in the model.

\begin{tabular}{|l|l|l|l|l|}
\hline \multirow{2}{*}{$\begin{array}{l}\text { Maximum number of arrows } \\
\text { pointing at a construct }\end{array}$} & \multicolumn{4}{|l|}{ Minimum $\boldsymbol{R}^{2}$ in the model } \\
\cline { 2 - 5 } & 0.1 & 0.25 & 0.50 & 0.75 \\
\hline 2 & 110 & 52 & 33 & 26 \\
\hline 3 & 124 & 59 & 38 & 30 \\
\hline 4 & 137 & 65 & 42 & 33 \\
\hline 5 & 147 & 70 & 45 & 36 \\
\hline 6 & 157 & 75 & 48 & 39 \\
\hline 7 & 166 & 80 & 51 & 41 \\
\hline 8 & 174 & 84 & 54 & 44 \\
\hline 9 & 181 & 88 & 67 & 46 \\
\hline 10 & 189 & 91 & 59 & 48 \\
\hline
\end{tabular}


Table 01 - Reduced version of the table presented by Hair et al., (2014) for estimating minimum sample size on "Minimum R-Squared Method".

Although the 10-times rule method is a simple application for the researchers, it has been depicted that inaccurate estimates (Goodhue et al., 2012). A smaller sample can be used with PLS-SEM when other methods are failed to make the analysis. However, the nature of the population directly affects the legitimacy of such analysis which depends on the heterogeneity of the population (Sarstedt, Ringle, \& Hair, 2017). Hence, a badly designed sample will be given wrong analysis by the PLS-SEM (Sarstedt et al., 2017). As mentioned by Marcoulides \& Chin, (2013) a power analysis that includes model structure expected effect sizes and the significance level should be applied to determine the necessary sample size.

Kock \& Hadaya, (2018) suggest two related new methods for determining the minimum sample size in PLS-SEM applications which based on mathematical equations neither methods do not employ the disadvantages of the above mentioned Monte Carlo simulations or on elements that make up the 10 times rule or the minimum Rsquared methods. The first method is called the "Inverse Square Root Method", which uses the inverse square root of a sample's size for standard error estimation. The second is called "Gamma-Exponential Method" which has been implemented the gamma and exponential smoothing function corrections for calculating the standard error estimation employed in the first method.

\section{Inverse Square Root Method:}

While the researchers are analysing the samples from the population in PLS_SEM it generates the path coefficients called $\beta$. Each of these path coefficients may have a standard error called $S$. As mentioned by Kock, (2015) and Weakliem, (2016) if it has been plotted the distribution of the ratio of $\beta / S$, it indicates the critical T ratio for a specific significance level. Cohen, (1988), Goodhue et al., (2012) and (Kock, 2015) explained the power of the test and it depicts the probability that the ratio of $|\beta| / S$ lies in greater than the critical T ratio for a given specific significance level chosen. $|\beta|$ is the absolute value which denotes the strength of the path coefficient influencing the power. The significance level normally used as in the researches is 0.05 or $(P<.05)$. Hence, the critical T ratio can be denoted as T.05. As well as generally in the researches, it is assumed that if the path coefficients are normally distributed, the power will be greater than 0.8. By using these properties Kock \& Hadaya, (2018) have presented the equation for calculating the minimum sample size as the positive integer which satisfies the following formula (1) under the inverse square root method

$N^{\wedge}>(2.486 /|\beta| \min )^{\wedge} 2$

However, the true standard error $S$ has been calculated using $S^{\wedge}$ and according to Kock \& Hadaya, (2018), it underestimates the corresponding true value at very small samples (i.e., $1<N \leq 10$ ), and overestimates it at greater sample sizes (i.e., $N>10$ ). Therefore, they have suggested the Gamma Exponential Method which has been introduced as a refinement of the inverse square root method which has explained by the following formula (2).

$|\beta| \min (\mathrm{Ne})^{\wedge} \wedge\left(\left((e|\beta| \min ) / \sqrt{ }\left(\mathrm{N}^{\wedge}\right)\right)\right)>2.486$

As with the gamma function correction equation, this equation can be solved with a computer program that starts with $\mathrm{N}^{\wedge}=1$ and progressive increments its value to 2,3 , etc. until the smallest positive integer that satisfies the equation is obtained.

\section{Summary and Conclusions}

The sample size determination of the SEM analysis is one of the most fundamental and crucial problems. The above review of the selection of minimum sample size determination in the prior literature has provided more than twelve methods that have been employed by the past researchers. At the beginning of 10 subjects per predictor rule mentioned by Nunnally, (1967) the debate started and various rules-of-thumbs have been 
introduced including ratio of indicators to latent variables by Boomsma, (1985) and Marsh et al., (1988), selecting sample size on various goodness of fit indices of SEM by Bearden, Sharma \& Teel (1982), Bentler, (1990), Bentler \& Bonett, (1980), Curran, West \& Finch (1996), Marsh et al., (1988), and Mulaik et al., (1989), N > 100 rule by Kline, (2005), 100 to 150 sample size by Ding et al., (1995), 10 times of the free number of parameters by Raykov, (2006). Ringle et al., (2012), mentioned it should be approximately eight constructs for a smaller sample. Monte Carlo simulation also has been employed as a method of determining the minimum sample size of the SME (Kock, 2016; Paxton et al., 2001; Robert \& Casella, 1999). A simple application such as "10 times the maximum number of inner or outer links pointing at any latent variable" used by the "10-times rule" (Hair et al., 2011) and it has been the method of more favourite in more researchers. Again the minimum $\mathrm{R}^{2}$ method also has been employed by Hair et al., (2014) it also more popular and has later been criticized in the literature. Finally, Kock $\&$ Hadaya, (2018) introduced the inverse square root method, and the gamma-exponential method and they proved those two appliances are fairly accurate than ever which the experiments has based on three Monte Carlo experiments.

According to past literature greater number of methods and rules-of-thumb have been employed to solve the fundamental issue of the minimum sample size determination of the SEM analysis. However, each of these methods contained its own limitations while applying the different models and hence, it's have been criticized in the literature. Basically, the foundation used to address the problem was the issue in most methods when the sample size determination is more critical on several factors that make influences to the final goodness-of-fit of the SEM analysis. Kock \& Hadaya, (2018) have addressed deeper on this nature of the problem and demonstrated more accurate and practical solutions to the problem. The first method is simpler and more attractive in its nature and the second method is much more complex than the first. The researcher who is not methodically rich can use these two methods more simply with small computer applications on both normal and non-normal data.

This paper provides more than twelve sample size determination methods and their contributions to the problem, lessons learned and their advantages and disadvantages in the past literature as a more nontechnical review. It is one of the contributions of this review. Most of the researchers who do not have the methodological and technical knowledge, use SEM as their analysis method face this fundamental and critical problem and this review provides the avenue to come up to the solution which they can find out how they should determine the sample size for their analysis based on SEM.

\section{References}

1. American Psychological Association. (2009). Publication Manual of the American Psychological Association, 6th Edition ( $6^{\text {th }}$ ed.). American Psychological Association.

2. Anderson, J. C., \& Gerbing, D. W. (1984). The effect of sampling error on convergence improper solutions, and goodness of fit indices for Maximum likelihood conformity factor analysis. Psychometrika, 49, $155-172$.

3. Bentler, P. M. (1990). Comparative fit indexes in structural models. Psychological Bulletin, 107(2), 238246. https://doi.org/10.1037/0033-2909.107.2.238

4. Bentler, P. M., \& Bonett, D. G. (1980). Significance tests and goodness of fit in the analysis of covariance structures. Psychological Bulletin, 88(3), 588-606. https://doi.org/10.1037/0033-2909.88.3.588

5. Boomsma, A. (1985). Non- convergence, improper solutions, and starting values in LISREL maximum likelihood estimation. Psychometrika, 50, 229-242.

6. Cohen, J. (1988). Statistical power analysis for the behavioral sciences ( $2^{\text {nd }}$ ed.). L. Erlbaum Associates. 
7. Ding, L., Velicer, W. F., \& Harlow, L. L. (1995). Effects of estimation methods, number of indicators per factor, and improper solutions on structural equation modeling fit indices. Structural Equation Modeling: A Multidisciplinary Journal, 2(2), 119-143. https://doi.org/10.1080/10705519509540000

8. Fornell, C., \& Bookstein, F. L. (1982). Two Structural Equation Models: LISREL and PLS Applied to Consumer Exit-Voice Theory. Journal of Marketing Research, 19(4), 440-452. https://doi.org/10.2307/3151718

9. Goodhue, D., Lewis, W., \& Thompson, R. L. (2006). PLS, Small Sample Size, and Statistical Power in MIS Research. Proceedings of the 39th Annual Hawaii International Conference on System Sciences (HICSS'06). https://doi.org/10.1109/HICSS.2006.381

10. Goodhue, D., Lewis, W., \& Thompson, R. L. (2012). Does PLS Have Advantages for Small Sample Size or Non-Normal Data? MIS Quarterly, 36(3), 981-1001. https://doi.org/10.2307/41703490

11. Hair, J. F., Hult, G. T. M., Ringle, C. M., \& Sarstedt, M. (2014). A primer on partial Least Squares Structural Equation Modeling (PLS-SEM). Thousand Oaks, California: SAGE Publications.

12. Hair, J. F., Ringle, C. M., \& Sarstedt, M. (2011). PLS-SEM: Indeed a Silver Bullet. Journal of Marketing Theory and Practice, 19(2), 139-152. https://doi.org/10.2753/MTP1069-6679190202

13. Hox, J. J., \& Bechger, T. M. (1999). An Introduction to Structural Equation Modelling. Family Science Review, 11, 354-373.

14. Kline, R. B. (1998). Methodology in the social sciences. Principles and practice of structural equation modeling. Guilford Press.

15. Kline, R. B. (2005). Methodology in the social sciences. Principles and practice of structural equation modeling $\left(2^{\text {nd }}\right.$ ed. $)$. Guilford Press.

16. Kock, N. (2015). One-tailed or two-tailed P values in PLS-SEM? International Journal of E-Collaboration, 11(2), 1-7.

17. Kock, N. (2016). Non-normality propagation among latent variables and indicators in PLS-SEM simulations. Journal of Modern Applied Statistical Methods, 15(1), 299-315.

18. Kock, N., \& Hadaya, P. (2018). Minimum sample size estimation in PLS-SEM: The inverse square root and gamma-exponential methods. Information Systems Journal, 28(1), 227-261.

19. Kock, N., \& Lynn, G. S. (2012). Lateral collinearity and misleading results in variance-based SEM: An illustration and recommendations. Journal of the Association for Information Systems, 13(7), 546-580.

20. MacCallum, R. C., \& Austin, J. T. (2000). Application of structural equation modeling in psychological research. Annual Review of Psychology, 51, 201-226.

21. MacCallum, R. C., Widaman, K. F., Zhang, S., \& Hong, S. (1999). Sample size in factor analysis. Psychological Methods, 4, 84-99.

22. Marcoulides, G. A., \& Chin, W. W. (2013). You write, but others read: Common methodological misunderstandings in PLS and related methods. In New perspectives in partial least squares and related methods (pp. 31-64). Springer, New York, NY.

23. Marsh, H. W., Balla, J. R., \& McDonald, R. P. (1988). Goodness-of-fit indexes in confirmatory factor analysis: The effect of sample size. Psychological Bulletin, 103(3), 391-410. https://doi.org/10.1037/0033-2909.103.3.391 
24. Miaoulis, G., \& Michener, R. D. (1976). An Introduction to Sampling. Kendall/Hunt Publishing Company: Dubuque, lowa.

25. Mulaik, S. A., James, L. R., Van Alstine, J., Bennett, N., Lind, S., \& Stilwell, C. D. (1989). Evaluation of goodness-of-fit indices for structural equation models. Psychological Bulletin, 105(3), 430-445. https://doi.org/10.1037/0033-2909.105.3.430

26. Muthén, L. K., \& Muthén, B. O. (2002). How to Use a Monte Carlo Study to Decide on Sample Size and Determine Power. Structural Equation Modeling: A Multidisciplinary Journal, 9(4), 599-620. https://doi.org/10.1207/S15328007SEM0904_8 Nunnally, J. C. (1967). Psychometric theory. McGrawHill.

27. Paxton, P., Curran, P. J., Bollen, K. A., Kirby, J., \& Chen, F. (2001). Monte Carlo experiments: Design and implementation. Structural Equation Modeling, 8(2), 287-312.

https://doi.org/10.1207/S15328007SEM0802_7

28. Raykov, T. (2006). A first course in structural equation modeling ( $2^{\text {nd }}$ ed.). Mahwah, NJ: Lawrence Erlbaum Associates.

29. Ringle, C. M., Sarstedt, M., \& Straub, D. W. (2012). Editor's comments: A critical look at the use of PLSSEM in MIS quarterly. 36(1). https://doi.org/10.2307/41410402

30. Robert, C., \& Casella, G. (1999). Monte Carlo Statistical Methods. Springer-Verlag. https://doi.org/10.1007/978-1-4757-3071-5

31. Sarstedt, M., Ringle, C. M., \& Hair, J. F. (2017). Partial Least Squares Structural Equation Modeling (In: Homburg C., Klarmann M., Vomberg A. (Eds). Handbook of Market Research, Heidelberg: Springer.

32. Singh, A. S., \& Masuku, M. B. (2014). Sampling techniques \& determination of sample size in applied statistics research: An overview. International Journal of Economics, Commerce and Management, Vol II (Issue 11).

33. Tanaka, J. S. (1987). How big is enough? Sample size and goodness-of-fit in structural equation models with latent variables. Child Development, S8, 134- 146.

34. Velicer, W. F., \& Fava, J. L. (1998). Affects of variable and subject sampling on factor pattern recovery. Psychological Methods, 3(2), 231-251. https://doi.org/10.1037/1082-989X.3.2.231

35. Weakliem, D. L. (2016). Hypothesis Testing and Model Selection in the Social Sciences (1 edition). The Guilford Press.

36. Westland, J. C. (2010). Lower bounds on sample size in structural equation modeling. Electronic Commerce Research and Applications, 9, 476- 487. https://doi.org/10.1016/j.elerap.2010.07.003

37. Wilkinson, L. (1999). Statistical methods in psychology journals: Guidelines and explanations. American Psychologist, 54(8), 594-604. https://doi.org/10.1037/0003-066X.54.8.594

38. Wolf, E. J., Harrington, K. M., Clark, S. L., \& Miller, M. W. (2013). Sample Size Requirements for Structural Equation Models: An Evaluation of Power, Bias, and Solution Propriety. Educational and Psychological Measurement, 76(6), 913-934. https://doi.org/10.1177/0013164413495237 